\title{
À quelles conditions les lapsus clavis sont- ils des jeux de mots?
}

Résumé : L’article analyse les lapsus effectués avec un clavier d'ordinateur, dans un corpus de courriels, et soutient la thèse que certains d'entre eux, involontaires par définition, peuvent être considérés comme de bons lapsus et, à ce titre, comme des jeux de mots intentionnels. L'article fait d'abord le point sur les différents lapsus, linguae, calami et clavis et revient sur la distinction entre lapsus, coquille ou erreur. Il tente ensuite de hiérarchiser des critères, morphologiques, syntaxiques, sémantiques, interactionnels qui accompagnent les lapsus au fil du discours, afin d'expliquer les jugements de plus ou moins grande réussite des lapsus, en tant que jeux de mots. Il distingue notamment les critères morphologiques et phonologiques qui sont l'indice d'un énoncé problématique ; les critères sémantiques qui permettent de pointer vers l'hypothèse lapsus; les critères énonciativo-interactionnels qui ratifient l'hypothèse et apprécient le lapsus; le critère de pertinence/réussite qui dépend de la partageabilité du lapsus. La hiérarchisation de ces critères n'est pas seulement un fait de production, mais de réception, mettant en avant la coconstruction des critères de réussite, qui transforment un lapsus a priori involontaire en une création a posteriori intentionnelle. Enfin, comme le lapsus repose sur un conflit conceptuel, l'article en propose une analyse pragma-énonciative, afin de rendre compte du conflit, des phénomènes de prise en charge des points de vue cumulatifs ou substitutifs, en lien avec des postures de co-, sur- et sous-énonciation.

Mots clés : co-énonciation, conflit conceptuel, écoute, jeu de mots, lapsus, lapsus clavis, points de vue (cumulatifs, substitutifs), prise en charge, sousénonciation, sur-énonciation

\section{Introduction}

Je voudrais analyser les lapsus (Lp) effectués avec un clavier d'ordinateur, dans un corpus de courriels, et soutenir la thèse que certains d'entre eux, involontaires par définition, peuvent être considérés comme de bons Lp et, à ce titre, comme des jeux de mots (JDM). Le corpus recueilli se compose d'une petite centaine de mails professionnels, recueillis entre 2014 et 2016 (pour l'essentiel). Dans leur quasi totalité, ce sont des mails que j'ai envoyés ou reçus, excepté quelques uns 
qui m’ont été transmis par des collègues. Ce corpus se caractérise par ses dimensions auto- et/ou hétéro-dialogiques, les lapsus faisant l'objet d'un commentaire éventuel de leur auteur, auquel s'ajoute celui du destinataire. Je laisserai de côté la question des Lp dus aux logiciels de correction automatique qui modifient le texte à l'insu du scripteur. La prise en compte de ce paramètre ne modifierait cependant pas mon analyse, car certaines modifications involontaires peuvent être récupérées et réinterprétées par l'énonciateur/écouteur (ou les lecteurs), si elles font sens, dans le co(n)texte ou selon le genre. Je ferai d'abord le point sur les différents Lp, linguae, calami et clavis (2), passerai rapidement sur la distinction entre Lp, coquille ou erreur (3), avant de hiérarchiser des critères, phonétiques, morphologiques, syntaxiques, sémantiques, interactionnels, afin d'expliquer les jugements de plus ou moins grande réussite des Lp, en tant que JDM (4). Enfin, je proposerai de la condensation caractéristique des Lp une analyse pragma-énonciative en termes de conflits de points de vue (PDV) cumulatifs ou substitutifs, en lien avec des postures de co-, sur- et sousénonciation (5). ${ }^{1}$

\section{Lapsus linguae, calami, clavis}

Les Lp linguae correspondent au fait de prononcer un mot ou une forme $\mathrm{e}^{2}$ pour un(e) autre (Fenoglio 2003 : 64), avec une rupture d'isotopie et / ou du continuum discursif. La survenue d'une forme inattendue s'accompagne souvent de silence, « d'un arrêt brusque du trajet énonciatif », de reprises et de commentaires, signalant une « infraction de la parole singulière dans le discours » (Fenoglio 1997 : 42). Le Lp est donc « suffisamment marqué discursivement pour être sentendu» par l'un ou l'autre des protagonistes de l'interénonciation, sans cependant que la compréhension de son advenue soit donnée; il trouble l'ordre énonciatif annoncé, mais est très vite rapporté à un «en dehors` » (Fenoglio 1997 : 46).

L'expression de $L p$ linguae date du XIX ${ }^{\mathrm{e}}$ siècle. Par comparaison, celle de $L p$ calami, est attestée dès le XVII ${ }^{\mathrm{e}}$ siècle en français, invoquée par les spécialistes qui établissent des textes anciens, traquent coquilles ou Lp, tant du point de vue de l'établissement du texte que de son interprétation. Peut-être est-ce cette activité de clerc qui explique la moindre diffusion de la notion et de l'expression.

1 Les notions de JDM, PDV (cumulatif ou substitutif) et de postures énonciatives seront définies infra au moment où elles seront convoquées.

2 Selon Rossi et Peter-Defare (1998 : 24-37), 30 \% des Lp sont des mots entiers, 5 \% des syllabes, $65 \%$ des phonèmes. Ce calcul est indicatif, vu la fréquence des monosyllabes en français. 
Quant à la notion / dénomination de Lp clavis - comme aussi la dénomination de Lp tapoti ${ }^{3}$-, elle est la plus récente des trois, et renvoie non plus au Lp qui serait fait avec le calame, mais avec le clavier. ${ }^{4}$ Cependant, bien que l'usage de ce dernier se soit fortement répandu, il n'a pas entrainé une attention particulière pour cette forme, plus souvent repérée comme erreur ou coquille que comme un jeu sur et avec les mots / formes.

Je fais l'hypothèse que, par-delà des différences liées au canal ou à une technologie, il y a une constellation de phénomènes convergents. Je restreins mon analyse au français et au clavier AZERTY - dont je reproduis en annexe la disposition des touches -, mais ses résultats sont généralisables, mutatis mutandis, à d'autres langues et d'autres types de clavier. Compte tenu de cette hypothèse, je partirai des Lp linguae pour investiguer les Lp clavis.

\section{Coquilles, erreurs ou lapsus ?}

Perdue (1980) distingue les Lp linguae et calami des erreurs de performance transitoires de l'apprenant. Parallèlement, il y a le continent des pratiques irrégulières des locuteurs experts, considérées comme des coquilles ou des erreurs de saisie (des fautes de frappe, comme on hésite à dire aujourd'hui, sous l'influence des didacticiens qui mettent en garde contre les formulations stigmatisantes / culpabilisantes). Il y a ainsi des erreurs de saisie en (1), une coquille en (2), en l'absence d'intention et de signification décelables :

(1) Tout cela aide à définir le type de réalité om se meut le jeu : c'est une réaité lystique et qui emprunte au sacré quelques-uns de ses caractères les plus apparents ${ }^{5}$

(2) L'aboutissement meurtirer des logiques totalitaires (Dulong, Le témoin oculaire 1998 : 16)

3 La pensée du discours, Onglet «Énoncés mémorables », rubrique « Coquilles, lapsus et inventions ». Ainsi dans « Je fais un fion pas possible» [foin]. Dans tout l'article, j'adopte les conventions suivantes : le Lp est graissé, les éventuels auto-commentaires qui l'accompagnent sont en italique et soulignés, les hétéro-commentaires sont en italique et non soulignés. Les formes entre crochets, qui décodent le Lp ou apportent des informations nécessaires à sa compréhension, sont données par l'analyste, en dehors de l'interaction.

4 Lacan parle d'un Lp « orthographique » dans une lettre, sans qu'on sache s'il a été écrit à la main ou avec un clavier (Lacan 1975 : 27).

5 La version correcte est : « Tout cela aide à définir le type de réalité où se meut le jeu : c'est une réalité mystique et qui emprunte au sacré quelques-uns de ses caractères les plus apparents ». 
Ces altérations sont jugées non significatives. D’ailleurs, elles ne s'accompagnent d'aucune des manifestations évoquées ci-dessus.

Toutefois, pourquoi certaines altérations sont-elles jugées pertinentes par rapport à un vouloir dire projeté ou attendu $?^{6}$ Certes, il y a des risques de normativité à prétendre dire sans précautions quels sont les véritables Lp. Néanmoins, chacun a fait l'expérience de Lp jugés plus riches de sens que d'autres : ainsi, le Lp prononcé en 1975 à l'Assemblée nationale par le député Robert-André Vivien, à propos d'un projet de loi contre la pornographie, invitant ses confrères à « durcir leur sexe »/[leur texte], sera jugé plus significatif, plus drôle que «ma

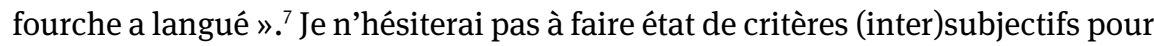
l'interprétation et l'appréciation des Lp et j'essaierai d'éviter le piège du normativisme en distinguant des critères de la zone proximale des Lp clavis, de ceux qui s’en éloignent.

\section{Les caractéristiques des lapsus}

\subsection{Caractéristiques générales des lapsus (linguae)}

Il existe maintes typologies des Lp : je citerai ici celles de Fenoglio (1997), de Rossi et Peter-Defare (1998), pour le français, d'Arnaud (1999), pour l'anglais, sans compter les travaux de Hockett (1984), Fromkin (1973), pour ne citer qu'eux. Je restitue le classement de Fenoglio, assorti de quelques exemples.

1. Du point de vue de l'analyste

1.1. En langue (rupture morpho-syntaxique) : «C'est un peu bizarre parce que pour moi il y a une espèce de, de deux allemands quoi, l'allemand de ma [la] guerre qui raconte sur des trucs que j'ai vu sur les nazis » (Fenoglio 1997 : 54);

1.2. En discours (perturbation de la chaîne parlée) : « ... oui alors ça je voulais donc, les relations avec les enfants, je ne parle jamais allemand, je ne parle que français avec mes allemands euh avec mes enfants » (Fenoglio 1997 : 56) ;

2. Du point de vue du locuteur

2.1. Lapsus entendu : en langue (pas forcément) ou en discours (toujours) ;

6 Ce vouloir dire est reconstruit attentionnellement sur la base des données fournies par l'énoncé, les attentes suscitées par le préconstruit et les prédiscours.

7 Voir le point de vue rafraîchissant de Culioli (2012 : 149) qui regrette que le linguiste hésite à formuler des jugements de valeur. 
2.1.1. Avec marqueur de correction et correction : «Ouais, ch'sais pas moi, a ce qui paraît c'est mieux, mais ch'sais pas si c'est plus dur ou pas assez dur, euh moins dur » (Fenoglio 1997 : 58) ;

2.1.2. Sans marqueur de correction : «C'est un peu bizarre parce que pour moi il y a une espèce de, de deux allemands quoi, l'allemand de ma [la] guerre qui raconte sur des trucs que j'ai vu sur les nazis » (Fenoglio $1997: 54)$;

2.2. Lapsus non entendu : « à partir du CM1 tout le monde fait de l'allemand mais c'est encore très superlatif on n'apprend pas grand-chose » [superficiel] (Fenoglio 1997 : 53).

Rossi et Peter-Defare (1998) dégagent six caractéristiques du Lp, sans s'arrêter sur la source du Lp ni sur les manifestations épi-, métalinguistiques ou métadiscursives qui retiennent l'attention de Fenoglio :

1. Interversions : « Le chour est faud »; [le four est chaud] : voir, sur les contrepèteries, Rabatel (2015a), et les contributions d'Attardo et de Braun et Schmiedel (in The Dynamics of Wordplay 6).

2. Omissions : " La proposition de geler [le salaire des] les fonctionnaires »;

3. Substitutions : "La mise en pièce... la mise en scène de la pièce ";

4. Insertions : «Il faut changer votre groupe de sécurité sociale, euh ! de sécurité » : [insertion de la collocation sécurité sociale] ;

5. Amalgame : «Un monde de chanson ébouristouflant » : [mot-valise réunissant ébouriffant (i.e. " décoiffant », « original ») + époustouflant] ;

6. Haplologie : «Non, il n'a pas été ambudgé... amputé le budget : [l'amalgame est favorisé par l'amuïssement de phonèmes aux sonorités équivalentes ou proches /pyt/ et /byd/].

À la différence des critères précédents, Arnaud (1999) propose une typologie qui, tout en reprenant des paramètres morpho-phonologiques et syntaxiques, privilégie la dimension sémantique :

1. Mots qui se ressemblent formellement (serviette/fourchette) ou ressemblance syntagmatique (« he rotates it by one quarter of a century [il le fait tourner d'un quart de siècle] er, of a turn [euh, de tour] ») ;

2. Mots apparentés sémantiquement (napkin $=$ serviette $[$ necktie $=$ cravate]) ;

3. Lapsus par contamination (par une pensée obsédante, un mot juste entendu, $\mathrm{vu}$, le contexte), erreurs qui sont précieuses pour penser le fonctionnement cognitif du langage. 


\subsection{Caractéristiques complémentaires à l'aune des lapsus clavis en discours}

Les caractéristiques ci-dessus concernent par principe les Lp clavis. Mais si l'on s'intéresse de près à ces formes écrites typographiques, on pourrait dégager d'autres traits phonologiques ou graphiques, morphologiques, syntagmatiques, ou technologiques, ces derniers renvoyant à la disposition des touches sur le clavier AZERTY (4.2.1 à 4.2.3), ainsi que des phénomènes textuels/interactionnels, (4.2.4) avec la manifestation de commentaires, leur emplacement, leur éventuelle succession.

\subsubsection{Nature du fragment-intrus}

Une première caractéristique complémentaire concerne la nature du fragmentintrus, qui peut correspondre à une lettre/un son, à une syllabe, à plusieurs, voire à un mot (voir le cas 4 de Rossi et Peter-Defare 1998, et, plus largement, leur typologie : voir supra, note 2).

\subsubsection{Place du fragment perturbateur par rapport au mot de base}

Une deuxième caractéristique concerne la position du fragment perturbateur par rapport au mot de base (caractéristique qui fait écho aux cas 1 et 4 de Rossi et Peter-Defare 1998) :

- Position à l'initiale comme en (3) et (4) :

(3) Chère Badyne [chère Nadyne] (04 mars 2015 $)$

(4) mes « charges daministratives » m'ont retardée comme elles me retardent sur tout, j'espère qu'il est encore temps (16 juin 2014) [administratives]

- Position finale, comme en (5):

(5) Je t'embrase [Je t'embrasse]

8 Compte tenu des conditions de collecte du corpus, les exemples ont été anonymisés autant que possible. 
- Position intermédiaire, comme le $n$, en (6), qui correspond à une glose de (4) sur laquelle je reviendrai :

(6) Ou « damn- istratives »; ou l'on est comme un damné à 100 fois remettre sur l'ouvrage... Pour rien!

_ Position antépénultième, comme dans l’amalgame « relectueurs » :

(7) J'ai pris à cœur mon rôle de relectueur oh le lapsus ! Je laisse, il est " joli ». C'est relecteur que je voulais dire, bien sûr !'

\subsubsection{Mécanisme morpho-phonologique et technologique de l'altération}

Une autre caractéristique concerne le mécanisme morpho-phonologique et, surtout, technologique de l'altération, compte tenu du clavier AZERTY :

- Touches contiguës : N/B (3), E/R (8), C/V (9), I/ O (10), I/ U (11), O/P (12), $\mathrm{P} / \mathrm{O}$ (13), $\mathrm{X} / \mathrm{C}$ (14) - ces trois derniers exemples relevant d'un amalgame selon Arnaud (1999) - :

(8) Je suis en Savoir/[en Savoie, Lapsus repéré par mon interlocuteur]

(9) de vice voix / [de vive voix]

(10) Je bisse (tiens, j’avais écrit je bosse, $X$ comprendra le lapsus calami) (04 février 2015)

(11) J'illustre brièvement la diversité des mouvements empathiques qui portent le narrateur à se mettre à la place de ses personnages ou de son lecteur, en jouant sur des points de vie $^{10}$ très différents, sans que, pour autant, le narrateur ne fasse parler directement ses personnages

9 Mail adressé au responsable du comité scientifique de Langue française, accompagnant ma première relecture en tant que membre du comité scientifique de la revue.

10 Le Lp est assorti de la note suivante, qui correspond à un auto-commentaire in praesentia (voir infra, 4.2.4) : «C'est bien évidemment point de vue que je voulais écrire. Mais la main a ses raisons... que la raison ne peut pas totalement ignorer. Comme tout lapsus, le lapsus calami éclaire sous un jour particulier une notion, et un rapport à la notion. Aussi ne souhaité-je pas corriger ce premier jet sans avoir marqué d'un petit caillou ce qui m'apparait comme une certaine vérité de la notion de point de vue : quelque chose qui engage un rapport à la vie, allant bien au-delà d'une pure et simple notion linguistique et narratologique - j'y reviendrai en conclusion. » (in Rabatel, version intermédiaire du texte paru dans Arborescences 6, Rabatel 2016a). 
(12) je vous envoie en pièce jointe les extraits d'un poème qui vient d'être oublié [publié] dans une revue poétique en ligne (territoires sauriens) (05 janvier 2016)

(13) J'ai publié de vous dire : parmi les colloques, il en est un d'important, c'est celui du CMLF [à une doctorante, 18 février 2016]

(14) tout cela mériterait d'être analysé, et les variables prises en compte selon les intervenants comme selon les sites, en distinguant la nature idéologique du site de son architexture [architecture] de ce qu'il permet ou non de faire, en termes d'analyse de la légitimité énonciative (24 avril 2016, rapport d'expertise)

- Suppression de touche comme S en (5), le T de (15) tueur accompagnant les enseignants stagiaires $^{11}$ (plutôt que les tuteurs), ou encore sevice compris (pour service compris) $^{12}$ :

(15) tueurs [tuteurs] censés accompagner les enseignants stagiaires

- Permutation/interversion $/ \mathrm{ia} / \rightarrow / \mathrm{ai} /{ }^{13}$, en (16), comme plus haut en (4), $\mathrm{AD} / \mathrm{DA}$ :

(16) C'est fiat [fait] cher X !

Allons bon, le lapsus calami. Si on disait fiat et que c'était sitôt fait, la vie serait belle!

- Adjonction, comme le $/ \mathrm{u} /$ en (7) qui transforme le relecteur en "relectueurs »/ relecteur, le /n/ de damnistrative en (4), le /r/ de « contreprèterie » en (17) :

(17) Les contreprèteries [contrepèteries] ont une dimension critique.

De fait, il en est beaucoup qui prennent des religieux pour cible!

De même le /1/ de (18), ou le /t/ de (19) :

11 Mail de l'ESPE de Lyon.

12 Mail de la mairie de Lyon, 2015.

13 Le phénomène vaut pour l'écrit. Mon commentaire écrit intégrant la dimension orale et multicodique, les mécanismes sont plus complexes, car la dimension multicodique entraine une bizarrerie syntaxique. Mais l'interprétation du JDM n'est pas à la syntaxe près, dès lors que le déplacement/rapprochement associatif produit une interprétation riche de sens (Aquien 2016 : 189-190). 
(18) J'ai défendu, en explosant [exposant] mon cadre théorique $\left(N D L R^{14}\right.$ : je laisse le lapsus juste pour vous amuser), une conception de la linguistique qui ne se désintéresse pas des conceptions énonciatives [version de travail d'une conclusion de thèse]

(19) J'ai acheté des trompettes de la mort pour ton trepas d'anniversaire [dit une épouse à son mari, pour son repas d'anniversaire] (exemple d'E. Danblon)

L'ajout relève bien du $L p$ calami parce que /p/ et /1/ sont contigus, approximativement l'un au dessous de l'autre, sur le clavier. ${ }^{15}$ Il n'en va pas de même en (20) dans la mesure où /e/ et /a/ ne sont pas contigus sur le clavier, mais sont séparés par une lettre.

(20) Cher X,

Nous pourrions $\boldsymbol{a f f e c t i v e m e n t ~ n o u s ~ r e ́ u n i r ~ d e m a i n ~ e n t r e ~} 14 \mathrm{~h} 30$ et $16 \mathrm{~h}$ dans mon bureau, soit le 4210.

Est-ce que cela te convient ? Bien cordialement, Y (exemple de C. Gérard)

\subsubsection{Manifestations discursives / interactionnelles accompagnant le Lp}

Une quatrième caractéristique complémentaire concerne les manifestations discursives / interactionnelles ${ }^{16}$ accompagnant le Lp, patentes si on prend en compte l'insertion de ce dernier dans le fil du discours ; elles ne se bornent pas aux marqueurs de correction relevés par Fenoglio mais intègrent aussi des gloses/commentaires.

- Présence d'un ${ }^{17}$ hétéro-commentaire (6) puis d'un auto-commentaire (21) in praesentia (même si c'est en différé vu le médium). Je précise que (4) con-

14 En l'occurrence, il s'agit d'une note (d'un commentaire) de la rédactrice.

15 Le critère de la contiguïté des lettres sur le clavier doit être relativisé par la dimension fonctionnelle. Une personne écrivant sur machine avec ses dix doigts utiliserait l'annulaire et l'auriculaire, comme le remarque Esme Winter-Froemel ; il n'en reste pas moins que le doigt (quel qu'il soit) peut, par inadvertance, déraper sur la touche contigüe.

16 Cette interaction peut orienter des échanges (voir (4)-(6) et (21), en situation de communication en face à face ou différée, dans les mails. Y a-t-il «interaction` lorsque nous lisons des JDM d'un locuteur absent ou d'un auteur des siècles passés? Le récepteur peut «interagir» avec le texte, selon les intentions qu'il prête à son auteur ; mais la réciproque n'est pas vraie. Ces interactions〉 sont secondaires, partielles, asymétriques, différentes par nature des interactions orales, collaboratives par définition - même si elles sont plus ou moins collaboratives. En revanche, d'autres interactions sont possibles, avec des tiers qui se positionnent par rapport à l'interprétation du récepteur, qu'ils confrontent avec les leurs ou celles qu'ils imputent à l'auteur.

17 Il est bien sûr possible qu'il y ait un ou plusieurs auto- ou hétéro-commentaires. 
cerne un rapport pour la promotion d'une enseignante-chercheure, auquel je réponds en (6), la locutrice de (4) répliquant en (21) :

(4) mes «charges daministratives» [administratives] m'ont retardée comme elles me retardent sur tout, j'espère qu'il est encore temps (16 juin 2014)

(6) Ah, le lapsus calami (ou le mot-valise) : "charges daministratives": charges administratives des dames?

Ce qui entraine derechef une réponse de la collègue :

(21) oui !!!

Ou « damn-istratives »; ou l'on est comme un damné à 100 fois remettre sur l'ouvrage... Pour rien! (16 juin 2014)

On voit apparaître ici une structure d'échange, avec un tour initial, sa réaction et la réponse à la réaction, qui vient clôturer cette micro-séquence, le tour de clôture ajoutant un /n/, mis en valeur par le tiret signalant cet ajout permettant de jouer avec le mot français damné, engendrant ainsi l'auto-commentaire final. L'interaction/l'interprétation ne s'attarde pas sur l'interversion $\mathrm{du} / \mathrm{d} / \mathrm{et} \mathrm{du} / \mathrm{a} /$, elle prend d'emblée au sérieux une formulation incongrue et cherche à trouver du sens, voire un surcroît de pertinence au point de vue $(\mathrm{PDV})^{18}$ projeté, qui visait à mettre en avant le caractère dévoreur de temps des tâches administratives, évoquées avec le substantif à l'axiologie négative « charges » plutôt qu'avec le substantif activités, usuel dans ce genre de littérature grise servant à l'évaluation des chercheurs. (6), tout en entérinant le PDV projeté / attendu de (4) - que je nommerai PDV2, infra - propose de ce qui est effectivement dit (PDV1), une glose en congruence avec la séquence phonétique [dam], en appui sur le substantif dame : les charges administratives sont souvent assumées par les femmes. À quoi l'auteure de (4), qui accepte cette hypothèse, en ajoute une autre, interlinguistique. ${ }^{19}$ Cette séquence n'est pas sans produire des effets, au plan argumentatif : car l'évocation conjointe de l'argument excusant le retard, reformulé sous sa variante sexiste ou sous ses caractéristiques axiologiques (travail de damné), justifie interactionnellement le retard, plaidant implicitement en faveur d'un examen bienveillant du dossier.

18 Voir infra 5. Pour une définition détaillée. Un PDV apparaît dans une prédication, et indique, par les choix de la référenciation, le point de vue de l'énonciateur sur l'objet, sans qu'il soit besoin de formuler un jugement explicite : voir Rabatel (2008a, 2008b, 2012b).

19 Comme en (16), en passant du français au latin. 
L'exemple (5') donne naissance, de même, à un hétéro-commentaire de la destinataire du message :

(5') Je t'embrase [Je t'embrasse]

[réponse immédiate de la destinataire, qui recite le message en l'assortissant d'une interjection amusée]

«Je t'embrase » : Waouh!

- Présence d'un auto-commentaire in praesentia ((10), (11), (16), (17), (18)) ou différé ((20'), (22), (23)), avec, en ce cas, la possibilité que l'auto-commentaire différé commente le Lp en s'adressant au destinataire du message (20') ou à une tierce personne ((22), (23)) :

(20’) Cher X,

Nous pourrions affectivement nous réunir demain entre $14 \mathrm{~h} 30$ et $16 \mathrm{~h}$ dans mon bureau, soit le 4210. Est-ce que cela te convient ? Bien cordialement, $Y$

[Premier mail suivi rapidement d'un deuxième avec un auto-commentaire rectificatif de $\mathrm{Y}$ ] : Merci de lire " effectivement "!!!

(22) Salut,

Mangeons-nous ensemble demain?

A bientpot

(rapporté par l'auteur à IF avec ce commentaire : Salut, pour la bonne bouche, ce mail que j'envoie à une collègue, et que tu sauras apprécier) (Fenoglio $2003: 72$ )

Fenoglio hésite entre faute de frappe et Lp et allègue l'absence d'accent circonflexe en faveur du Lp, avec un mot différent, le « pot ». La présence ou l'absence de l'accent relève d'une rationalisation secondaire comme le confirment l'autopuis l'hétéro-commentaire de (22) et (23) :

(23) La commission sera socio-SE \& Info-com.

Merci et à bienpôt.

(je te laisse cet heureux lapsus)

[...]

Cher M.

[...]

Par ailleurs le pot est samedi 10 mars de $11 \mathrm{~h}$ à $13 \mathrm{~h}$ et non lundi (il ne restera plus rien plus rien mon pauvre!)

Comme dans les exemples (4)-(6) et (21), le Lp suscite des commentaires en chaine, qui traitent du Lp comme d'un mot d'esprit, voire comme un JDM. 
- Commentaires métalinguistiques ou métadiscursifs ${ }^{20}$, hyperboliques, portant sur le contenu du Lp (Bravo !, Magnifique!, Super!, C'est le cas de le dire !), ou ironiques envers l'auteur du Lp (l'inconscient a encore frappé, Je ne te le fais pas dire, Tu nous en dira tant!), souvent accompagnés d'interjections (Hum hum, Ouh la là !, Waou ! (5)), Ben dis donc).

- Présence de signaux paralinguistiques, à l'oral, rires, clins d'œil, signes d'approbation (pouce dressé, applaudissements, etc.), critères auxquels il faut encore ajouter la direction de ces signaux, vers l'auteur du Lp ou des tiers. À l'écrit, les smileys sont l'équivalent iconique de signaux paralinguistiques, dans le même temps qu'ils jouent un rôle modalisateur (Halté 2013).

Ces interactions scellent la connivence du ou des récepteurs avec l'auteur, comme on le voit dans les exemples (4)-(6)-(21) et (22)-(23). Elles indiquent une réaction amusée et positive face au Lp, renforçant l'hypothèse que l'énoncé manifeste, par-delà sa dissonance, est apprécié pour sa ludicité et son surcroît de signification. L'hypothèse est confirmée par la rareté des réactions négatives ${ }^{21}$ envers un Lp sans grande signification (« ma fourche a langué »). En ce cas, le Lp fait l'objet d'une correction, sans manifestation théâtralisée, puisqu'il n'en vaut pas la peine.

Tels sont les critères plus spécifiques aux Lp clavis, qui se produisent à l'intérieur d'un mot. Les formes contiguës forment la zone proximale des Lp clavis ; les autres Lp relèvent d'une logique signifiante qui n'est plus aussi contrainte par la contiguïté des touches du clavier (14). De plus, ces formes ne font sens que si les autres critères sémantiques, co(n)textuels et interactionnels sont réunis : mais alors, on entre dans une autre zone proximale, celle des Lp réussis, candidats pour être de « bons » JDM, et qui nécessitent le plus souvent une certaine proximité (de tous ordres, j'y reviendrai) avec le locuteur : c'est pourquoi (9) parait « moins bon » que (10), moins bon aussi que (14), qui n'est pas dans la zone proximale. De même, bien que daministrative en (4) ou damnistrative en (21) n'appartiennent pas à la zone proximale, ils sont cependant des Lp clavis intéressants. Dès lors, se pose la question de savoir si l'on peut avancer des arguments rationnels et partagés pour rendre compte de la réussite des Lp et dégager des critères objectifs de proximité, voire de connivence.

20 Le lecteur trouvera dans ce volume d'autres exemples de commentaires (voir KerbratOrecchioni), pouvant éventuellement engendrer du texte, en étant au cœur du processus créateur (voir Poier-Bernhard, in The Dynamics of Wordplay 6).

21 Sauf si elles sont ironiques, mais en ce cas leur caractère antiphrastique revient à une stratégie d'approbation détournée. 


\subsection{Hiérarchiser les critères pour rendre compte du degré de réussite du lapsus : de l'intérêt du jeu avec la proximité et la distance}

Les critères précédents gagnent à être combinés, car il est impossible de rendre compte d'une réalité complexe à partir d'un seul niveau linguistique. En effet, toute interversion, omission, substitution, insertion, amalgame, haplologie ne donnent pas nécessairement naissance à des Lp - et il en va de même pour les critères listés par Arnaud ou Fenoglio. Il faut encore, outre la ressemblance formelle, que les mots soient apparentés sémantiquement ou qu'une contamination s'explique par des ressemblances formelles ${ }^{22}$, voire par la situation. Il faut aussi que le locuteur seul ou les récepteurs aient conscience du Lp, le manifestent par des corrections, commentaires ou réactions paralinguistiques. Pour qu'un Lp soit jugé « réussi », tout subjectif que soit ce critère, il faut que les critères sémantiques ci-dessus reposent sur deux autres critères complémentaires : que les mots se ressemblent au plan du signifiant (graphique plutôt que sonore) et surtout permettent de construire des points de vue, des prédications complémentaires sur l'objet, qu'elles soient (ré)interprétées comme pertinentes dans le co(n)texte, passé un premier trouble dû à la bizarrerie de la forme et à son incongruité par rapport au co(n)texte. Cela explique que les mêmes jeux morpho-phonologiques puissent être à peine remarqués dans un cas, et jugés de très jolis Lp dans d'autres, selon les co(n)textes. Ainsi, le Lp clavis « bienpôt », en (22), ne fait sens que parce que le pot est proche. Mais il m'arrive d'écrire bientpôt dans des co(n)textes où l'altération est perçue comme coquille. De même, il est fréquent que j'écrive contreprèterie pour contrepèterie, sans que cette coquille soit reconnue comme un Lp significatif, à l'inverse de (17), où le co-texte s'y prête.

Les critères ci-dessus fonctionnent solidairement, sans être de même niveau, car les critères sémantiques et énonciativo-interactionnels prédominent. Quant au critère de pertinence/réussite du Lp, qui analyse le Lp en tant que mot d'esprit, voire JDM, il est sanctionné par les réactions des récepteurs, mais aussi par celle du premier des récepteurs, le locuteur lui-même... Ce critère interactionnel doit bien sûr être complété par les critères sémantiques qui ratifient l'existence $\mathrm{du}$ Lp en tant que jeu de mot, jouant avec les situations (bientôt / bienpot), et avec la situation et la forme signifiante : (exposer/exploser, trepas/repas d'anniversaire, etc.). Si toutes ces conditions sont remplies, le Lp clavis est un JDM, puisque

22 Toutefois, le critère de la complexité formelle, analysé dans ce volume par Kerbrat-Orecchioni, n'est pas pertinent pour le Lp, car son caractère involontaire interdit toute recherche intentionnelle de la complexité. 
sa formulation, due au hasard, est jugée comme une réussite significative. Il faut encore ajouter que le critère pragmatique/interactionnel des réactions est luimême fonction d'une échelle de partageabilité : c'est pourquoi (4), en dépit de sa reconnaissance dans les échanges, est moins partageable par tous les lecteurs additionnels, que les Lp qui font immédiatement sens dans une communauté, comme cela est le cas pour architexture/architecture, relectueur/relecteur, contreprèterie/contrepèterie, tueur/tuteur, qui feront davantage sens pour des universitaires spécialistes de rhétorique, des formateurs d'enseignants que pour des locuteurs étrangers à ces communautés. Mais, ces communautés étant restreintes, le Lp sera moins partageable que le Lp de R. A. Vivien (durcir leur sexe/texte). À l'autre bout, je peux apprécier mon Lp je suis en savoir/Savoie, le destinataire qui me connaît aussi, mais c'est un private joke.

Par conséquent, le critère de pertinence dépend de la reconnaissance et de la partageabilité du Lp, ainsi que de son degré de réussite. Ce critère est épineux, la réussite étant une notion subjective. Cependant, le jugement de réussite peut s'argumenter : en règle générale, la réussite sera d'autant plus grande que le Lp sera reconnu, que sa signification sera jugée non anecdotique, profonde, amusante, en décalage par rapport aux normes linguistiques et éthiques, par rapport aux attentes, à la situation, au locuteur. Partant de là, il convient de mesurer que ce critère ne peut s'apprécier que par rapport à la prise en compte de données situationnelles. Il n'est par exemple pas indifférent que le Lp de (18) émane d'une doctorante, que celui de (20) s'adresse à une femme, et de même pour (4) et (6). C'est la raison pour laquelle certaines des explications évoquent ces données, en les ayant anonymisées, mais sans aller jusqu'à supprimer toutes les données situationnelles, comme certains relecteurs me l'ont demandé en alléguant des raisons éthiques. Ce souci est respectable, mais il peut s'avérer contre-productif dans ses excès. En l'occurrence, supprimer des données situationnelles au motif que la personne pourrait se reconnaître, c'est oublier que le sens ne saurait faire l'économie de telles données ; je m'en suis donc tenu à des données générales. Cependant, chacun sait que certains JDM ne sont bons non seulement parce qu'ils sont partagés, mais encore lorsqu'ils le sont sur le dos d'une innocente victime... Il y a là une contradiction, qu'il n'est au demeurant pas très difficile de résoudre quand les exemples n'attentent pas fondamentalement aux faces positive ou négative des personnes, comme c'est le cas dans mon corpus.

L'ensemble des remarques précédentes n'est pas sans faire écho aux travaux de Koch et Oesterreicher (1985, 2001) sur «l'immédiat communicatif » et la « distance communicative » (reformulés ci-après en proximité et distance). Ces auteurs listent un certain nombre de caractéristiques qui distinguent, d'une 
façon générale, la langue parlée (Sprache der Nähe) de la langue écrite (Sprache der Distanz) :

Tab. 1 : Caractéristiques de l'immédiat communicatif et de la distance communicative (Koch et Oesterreicher $2001: 586$ )

\begin{tabular}{lll}
\hline 1) communication privée & $v s$ & communication publique \\
2) interlocuteur intime & $v s$ & interlocuteur inconnu \\
3) émotionnalité forte & $v s$ & émotionnalité faible \\
4) ancrage actionnel et situationnel & $v s$ & détachement actionnel et situationnel \\
5) ancrage référentiel dans la situation & $v s$ & détachement référentiel de la situation \\
6) coprésence spatio-temporelle & $v s$ & séparation spatio-temporelle \\
7) coopération communicative intense & $v s$ & coopération communicative minime \\
8) dialogue & $v s$ & monologue \\
9) communication spontanée & $v s$ & communication préparée \\
10) liberté thématique & $v s$ & fixation thématique \\
etc. & & \\
\hline
\end{tabular}

Cependant, ces caractéristiques ne sont pas si homogènes que cela, certains genres et usages de l'oral ou de l'écrit mêlant proximité et distance ou distance et proximité : ainsi les mails relèvent-ils de la distance, mais les destinataires peuvent être proches par le partage de valeurs, de connaissances actionnelles, situationnelles, référentielles ((4), (22)), la communication peut être publique ((11), (15)) ou privée ((3), (5)), voire jouer avec cette distinction de principe dans le cas de destinataires multiples ((22), (23)). Certains mails peuvent reposer sur une coopération communicative minime ou intense ((6), (19), (23)), sur un ancrage actionnel et situationnel, voire un fort ancrage référentiel dans la situation ((8), (10)) ou au contraire reposer sur un détachement envers ces mêmes variables (5). Mais l'important n'est pas là, il réside plutôt dans le fait que le $L p$ bouleverse les attentes (et donc les codes qui leur sont liés) : ainsi une communication en principe dénuée d'émotionnalité se trouve-t-elle réinterprétée comme Lp significatif, chargé d'une forte émotionnalité, comme en (20); ainsi une thématique professionnelle par principe bienveillante devient-elle réinterprétée en un sens malveillant, comme en (7) et (15) ; ainsi une formule de politesse ou une communication strictement professionnelle sont-elles réinterprétées en jeux de séduction ((5), (20)), un repas d'anniversaire en l'honneur du conjoint est-il réinterprété en souhait de sa disparition (19), le respect qu'un doctorant doit prendre envers les cadres théoriques de ses prédécesseurs est-il lui aussi réinterprété 
négativement... Les Lp jouent donc de la proximité et de la distance, ils jouent avec cette opposition, et ce jeu fait partie du sentiment de réussite du Lp, tout en influant sur sa partageabilité. Cependant, un Lp n'a pas besoin d'être largement partagé pour être bon, comme le montre (8).

En résumé,

- les critères morphologiques et phonologiques sont l'indice d'un énoncé problématique ;

- les critères sémantiques pointent vers l'hypothèse Lp ;

- les critères énonciativo-interactionnels ratifient l'hypothèse le Lp et enrichissent son interprétation ;

- le critère de pertinence, qui dépend de la reconnaissance et de la partageabilité du Lp, influe sur son degré de réussite.

\subsection{Lapsus, jeux de mots et écoute}

On peut donc dire du Lp réussi, comme Quintilien le dit des figures, que « la figure de mots serait un défaut, si elle n'était pas voulue mais accidentelle $»^{23}$ (Institution oratoire [vers 93 apr. J.-C., Livre IX, 3, 2 ; 1978 : 202). L'intentionnalité, fût-elle de deuxième temps, réactive, pose la question du rapport du Lp au JDM. La réponse dépend de la définition du JDM (Lecolle 2016 ; Thaler 2016 ; WinterFroemel 2016). En un sens restreint, il y a JDM lorsque les mots jouent (intentionnellement ${ }^{24}$ ) avec les signifiants (phoniques et/ou graphiques) et avec les signifiés. Mais il peut aussi y avoir JDM, en un sens étendu, lorsque des mots / prédications bien formés jouent avec un seul des plans de l'expression ou du contenu et avec la situation. C'est le cas des énoncés ironiques, humoristiques, ou encore des énoncés hyperboliques : car c'est une autre façon de jouer avec le langage que s'amuser avec ses inappropriétés, ses incongruités, ses effets inattendus, dans une optique profondément "désacralisante » (Benveniste [1947] 2015 : 181). Le Lp relève du JDM au sens étroit (p. ex. (7), (15), (18), (19)) ou large (p. ex. (8), (22)).

N'y a-t-il pas cependant une contradiction entre le caractère souvent intentionnel des figures et celui, involontaire, des Lp ? La contradiction n’a pas lieu d'être car involontaire n'est pas synonyme d'inintentionnel. Ainsi, un Lp, involontaire par définition, peut cependant être considéré par le locuteur, ou, le

23 " Esset enim eiusmodi schema uitium si non peteretur, sed accideret».

24 Mais cette intentionnalité peut intervenir au moment de la production d'un énoncé comme à celui de sa réception. 
plus souvent, par les destinataires, comme intentionnel, en ce qu'il trahirait des significations cachées, intentionnelles, du moins au regard des problématiques de l'inconscient, pour lesquelles les actes manqués sont toujours volontaires, à un certain degré. Le jugement sur ce qui est involontaire et intentionnel ne renvoie donc pas nécessairement au même locuteur ou à la même position énonciative (ou point de vue, voir Rabatel 2012b) - comme on le verra mieux avec l'analyse pragma-énonciative des Lp qui condensent des conflits de points de vue, renvoyant à deux énonciateurs distincts, ou à deux positions énonciatives qui cohabitent dans la même voix du locuteur - mais il porte bien sur le même énoncé. La distinction entre involontaire et intentionnel requiert donc qu'on accorde une égale importance au locuteur et $\mathrm{au}(\mathrm{x})$ récepteur(s) et qu'on distingue les divers PDV qu'endosse le locuteur.

C'est en effet le récepteur, à la condition qu'il s'érige en co-énonciateur, qui fait ou parfait la créativité lexicale/verbale pleine et entière du Lp. Cette approche par le récepteur concerne aussi le producteur en tant qu'il est le premier récepteur de son dire. Parler de récepteur est une approximation, mieux vaudrait dire l'écouteur, au sens de «non pas s'écouter parler, mais s'écouter quand on parle» (Culioli, in Ducard 2004 : 20). Bravo (2011 : 198) cite les propos d'André Green (2003 : 47) à propos de ce qu'il nomme la « rencontre analytique » : « Cette rencontre est faite de parole et d'écoute $:^{25}$ écoute non de ce qui est dit mais de «ce qui se parle» dans ce qui est dit ». L'écoute peut être involontaire ou volontaire ${ }^{26}$, active ou « flottante ${ }^{27}$, mais toujours les sens en éveil... Enfin, l'écouteur est un actant syncrétique, qui peut englober le locuteur, qui en est d'emblée conscient ou qui reconnaît le Lp après que les autres écouteurs le lui ont fait remarquer, mais aussi les autres destinataires, que leurs réactions érigent en co-énonciateurs de PDV1. Il y a ainsi une dissymétrie entre le caractère imaginaire et projectif de l'image du locuteur/énonciateur à qui on prête un PDV attendu (PDV2) et le caractère syncrétique de la prise en charge de PDV1, par l'énonciateur et par les écouteurs en connivence avec lui.

25 On peut imaginer aussi que le Lp résulte d'un dialogue externe ou intériorisé aboutissant à un questionnement, voire à une sorte d'interpellation (Juranville $2010: 65$ ).

26 La psychologie distingue l'attention volontaire, active, de l'attention involontaire, réactive : « Nous pouvons dire, en général, que l'attention volontaire est orientée vers un contenu sélectif, et l'attention involontaire vers un contenu qui s'impose » (Reik 2002 : 155 apud Bravo 2011 : 229). 27 « Désassujettie des lois de la temporalité » (Bravo 2011 : 199) et de la linéarité. 


\section{Points de vue en confrontation et postures énonciatives dans les lapsus}

L'écoute porte sur le message, sur sa matérialité signifiante, sur les associations qu'elle autorise en termes de significations. Comme ces dernières ne tiennent pas seulement à l'interprétation des altérations, mais portent sur l'ensemble de la prédication, elles requièrent une analyse énonciative.

\subsection{Locuteur, énonciateur, point de vue}

Je distingue, d'après Ducrot (1984), deux instances, le locuteur et l'énonciateur (Rabatel 2012b). ${ }^{28}$ Le locuteur est l'instance qui profère un énoncé, dans ses dimensions matérielles, phonétiques ou scripturales : c'est lui qui est la source du Lp. L'énonciateur correspond à une position (énonciative) qu'adopte le locuteur, dans son discours, pour envisager les faits, les notions, sous tel ou tel PDV. L'énonciateur est l'instance à partir de laquelle les contenus propositionnels sont agencés dans une prédication ${ }^{29}$, de façon à indiquer, en sus de la référenciation dénotative aux états du monde, le PDV du sujet sur le référent ainsi construit. C'est cette instance qui entre en jeu pour la prise en charge du Lp. J'emploie le terme de prédication parce que le PDV, autrement dit le processus de construction du sens, requiert d'emblée une analyse des prédications et de leur mise en texte, car c'est le niveau où les relations entre composants s'élaborent - selon leur polysignifiance (Lecolle 2007) et leur plurisémie (Nemo 2014), du signe à l'énoncé, accordant une grande importance sémantique au global (Rastier 2014 : 24-26), sans pour autant réduire la part du local (Rabatel 2015c : 136-139) -, pour désambiguïser ou au contraire jouer avec l'ambiguïté. ${ }^{30}$ C'est ainsi que le Lp est

28 La majuscule, suivie du chiffre 1, code le locuteur/ énonciateur primaire, dont le rôle domine les locuteurs / énonciateurs seconds (12/e2). La barre oblique indique le syncrétisme de L1 et de E1 ou de 12 et de e2. On code e2 seul, en l'absence d'acte de parole (Rabatel 2012a : 28).

29 Un PDV correspond le plus souvent à une prédication. Mais l'empan peut varier : d'une part, il peut englober plusieurs prédications de même thème ou co-orientées ; d'autre part, il peut se limiter à une lexie suffisamment marquée pour indiquer un PDV, a fortiori si elle est une formule (Krieg-Planque 2009).

30 Sur les marques linguistiques du PDV, voir Rabatel (2012b : 25-26). Insister ainsi sur les prédications et des situations de discours, c'est reconnaître qu'une partie de l'argumentation est dans la langue (Nemo 2016 : § 32-33), dans les lexies, mais qu’une autre lui échappe, parce que les mots sont soumis à des ajustements incessants, prélude à des processus de lexicalisation, de grammaticalisation ou de pragmaticalisation (Rabatel 2016b). Lorsque Nemo (2016 : § 150-154), 
bien produit par un locuteur, mais qu'il fait entendre deux PDV différents, que l'on peut attribuer l'un à l'énonciateur premier E1 du PDV manifeste (PDV1), l'autre à une autre image de l'énonciateur premier (un autre de E1, que l'on peut coder E1'), qui renvoie au PDV attendu (PDV2) que les écouteurs reconstruisent sur la base des instructions/contraintes du texte, des préconstruits ou prédiscours.

\subsubsection{Répartition PDV1/PDV2}

Le PDV intenté ou attendu (PDV2), qui n'a pas été prononcé est cognitivement premier par rapport au PDV manifesté (PDV1). Mais cet ordre cognitif est différent de l'ordre du discours, et c'est cet ordre du discours que je privilégie. Bref, dans le Lp, PDV1 est donné d'emblée, mais sa forme, qui s'écarte du PDV attendu (PDV2), est d'emblée jugée ludique, amusante, voire pertinente, parce qu'elle brise des convenances, des normes, voire des tabous, qui sont toujours à l'arrièreplan du processus interprétatif. En fait, le débat pourrait être réglé si j'abandonnais la numérotation des PDV et m'en tenais à PDV manifeste (PDV1) et PDV atten$d u$ (PDV2). Mais comme j'utilise dans d'autres circonstances cette numérotation (Rabatel 2008b, 2012c, 2013a, 2013b, 2015a, 2015b, 2015c, 2018), je la maintiens pour garder à la théorie sa dimension unifiée, et aussi parce qu'il me semble juste d'accorder la primauté à ce qui est dit, dans l'ordre où cela apparait.

\subsubsection{Construction / extraction des PDV}

Le modèle cognitif élaboré par Prandi (2016 : 77-78), pour l'analyse de la métaphore peut être mis à contribution pour toute situation de conflit cognitif. La reconstruction de PDV1 s'appuie sur les relations sémantiques entre le cadre, correspondant largement au thème de la prédication qui oriente vers une interprétation attendue, et le foyer, apportant une information nouvelle, dans le rhème. Dans PDV1, il y a un effet inhabituel de sens, parce qu'il y a une rupture entre le cadre et le foyer, qui fait «figure » - selon un processus de rendement maximal des énoncés (Bonhomme 2005 : 70) - en ce qu'il s'écarte d'une formulation / représentation attendues, tandis que PDV2 essaie de reconstruire un PDV en imaginant un foyer davantage en congruence avec le cadre. Parfois c'est le

insiste sur le fait que le sens ne se réduit pas aux approches morphémiques, il ouvre la porte vers cette extension. 
cadre qui doit être mis en congruence avec le foyer, puisque le Lp peut intervenir dans le foyer (12') ou dans le cadre (13’) :

(12') PDV1 : tout écrivain craint d'être oublié/PDV2 : tout écrivain a l'espoir d'être publié.

(13’) PDV1 : il est nécessaire de publier pour faire une carrière universitaire (je m'adressai à une de mes doctorantes) / PDV2 : J'ai craint d'oublier de donner une indication essentielle (concernant la nécessité de publier) pour faire une carrière universitaire.

L'interprétation du conflit repose ainsi sur l'extraction de deux PDV. Selon Prandi (2016 : 77-78), elle s'appuie sur la mise en œuvre de deux autres concepts complémentaires, la teneur et le sujet subsidiaire. Ce dernier correspond au fait que chaque PDV fait sens pour un énonciateur donné, qui n'est pas le même dans PDV1 et PDV2 : ainsi PDV1 de (12) renvoie à l'image d'un écrivain éprouvant les affres de l'oubli, alors même qu'il a été publié, tandis que PDV2 ne fait sens que pour énonciateur écrivain qui aspire de tout son être à être publié. De même, PDV1 de (13) insiste sur l'absolue nécessité de publier, tandis que PDV2 renvoie plutôt à un énonciateur qui pourrait négliger (volontairement ou non) cette nécessité.

\subsection{Des points de vue en confrontation cumulatifs ou substi- tutifs}

Les PDV en confrontation reposent sur deux grandes relations sémantiques différentes : en (13), elles sont complémentaires, tandis qu'en (12) elles sont opposées : c'est cela qui permet de distinguer des PDV en confrontation cumulatifs, co-orientés, et d'autres, substitutifs, anti-orientés (Rabatel 2008b, 2012b, 2015c : 52-58).

Ainsi, il semble plus intéressant, plus significatif de comprendre qu'en (8), « je suis en savoir » (PDV1) condense le lieu de ma villégiature (je suis en Savoie, PDV2) et le fait que ce lieu de vacances est propice à des activités quasi monacales centrées autour du savoir (PDV1). PDV1 est ressenti par mon interlocuteur (et par moi aussi, à son invite) comme plus pertinent que le PDV factuel « je suis en Savoie » (PDV2). Ces PDV sont cumulatifs, l'un n'annule pas l'autre et cela explique que, les conditions de vérité de l'un n'enlevant rien aux conditions de 
vérité de l'autre, L1/E1 les prenne en charge ${ }^{31}$ tous les deux, même si PDV1 est plus pertinent.

Les PDV sont substitutifs lorsque, si PDV1 est vrai / positif, PDV2 est faux / négatif. Ces cas de figure sont assez rares dans les Lp. En effet, l'interprète peut considérer qu'en (7), disant relectueur, c'est finalement cette idée que les relectures ont une finalité tueuse, non empathique qui s'impose comme vraie - que j'aie voulu le dire ou non, peu importe. Mais rien ne permet de dire dans l'énoncé produit que la lecture substitutive l'emporterait : d'autant que, heureusement, tout chercheur a fait maintes fois l'expérience que les relecteurs n'étaient pas des tueurs ! De même pour " sévice compris ", " contreprètrerie » : les contrepèteries ne se moquent pas que du clergé, mais de tout ce qui est en principe objet de respect. D'ailleurs, le sel du JDM ne repose que sur la confrontation/condensation des deux PDV en une formulation (d)étonnante. La tendance dominante est celle des PDV cumulatifs ${ }^{32}$, en vertu du principe fondamental du double sens à faire entendre. Il existe malgré tout des Lp qui reposent sur des lectures substitutives plus nettes que (8), à l'instar de (10). La condensation « bosse » / « bisse », dans ce mail adressé au bureau d'une association, resté sans réponse, n'est-elle à prendre comme une sorte d'acte manqué (mais parfaitement réussi) qui trahit mes impatiences, quelque chose comme : si vous bossiez, je ne bisserai pas. Dans cette logique substitutive, les conditions de vérité de PDV2 ne sont plus valides. ${ }^{33}$

31 La notion de prise en charge (PEC) ne se limite pas à la conformité supposée de l'énoncé avec la vérité extralinguistique, elle s'appuie aussi sur l'adhésion à des valeurs, intersubjectives, évaluatives, axiologiques (Rabatel 2009). Je considère que c'est l'énonciateur - primaire (E1) ou secondaire (e2) - qui est la source de la PEC (effective pour E1, supposée pour e2) compte tenu de ses choix de référenciation du PDV. En contexte dialogique, E1 se positionne par rapport aux PDV qu'il attribue à des locuteurs / énonciateurs seconds, en manifestant explicitement (plus ou moins fortement) son accord, son désaccord ou en restant dans une attitude de "neutralité », se bornant à une prise en compte sans PEC (Roulet 1981 : 19 ; Rabatel 2009, 2012a, 2012b).

32 Cette remarque trouve un écho du point de vue psychanalytique : «Un des caractères des plus surprenants de cette logique [du signifiant, qui domine dans l'inconscient] est qu'elle met à bas les paires d'opposition que connaît le discours : positif/négatif (et donc présence/absence), actif/passif (et donc sujet/objet). Elle met sur le même plan une chose et son contraire. L'absence de contradiction dans l'inconscient n'a pas été la moindre des stupéfactions de Freud, quand il en a constaté le mécanisme dans le rêve. » (Aquien 2016 : 55).

33 Cette explication rationnelle n'empêche pas la pertinence d'une autre logique passant pardessus les contradictions : voir la note précédente. 


\subsection{Des postures énonciatives dans les lapsus}

Si l'on part de l'hypothèse que PDV1 est involontaire, on dira qu'il n'est pas $a$ priori pris en charge par l'énonciateur E1, même s'il a bien été prononcé par le locuteur L1, tandis que le PDV intenté serait celui que L1/E1 aurait voulu prendre en charge. Cependant, il arrive que L1, s'apercevant du Lp, l'entérine : il s'en fait donc a posteriori énonciateur aussi, comme le confirment les auto-commentaires de (7), (10), (16). Quant aux destinataires qui relèvent le Lp en l'appréciant, ils prennent aussi en charge PDV1, puisqu'ils le jugent plus pertinent que PDV2. ${ }^{34} \mathrm{La}$ sur-énonciation ${ }^{35}$ tient au fait que les commentaires qui suivent la prise de conscience du Lp entérinent sa pertinence inattendue, fût-ce sous une forme amusée, ludique, en ce sens que ce qui paraît incongru est interprété, comme une vérité profonde, cachée, éventuellement taboue, supérieure à PDV2: les exemples (7), (8), (10), (11), (18), (21) et (23), avec leurs commentaires, prennent en charge le surcroît de pertinence de PDV1. J'illustre cette sur-énonciation à partir des exemples (7) et (8) : écrire relectueur, plutôt que relecteur, et refuser de corriger, c'est accepter l'idée que le processus d'expertise peut relever parfois de l'assassinat. Ce rôle-là peut difficilement être « pris à cœur », en principe, mais il n'est pas écarté : plaisir d'un bon mot gratuit, d'une vérité qui n'ose se dire, c'est affaire d'interprétation. Ce qui importe, au plan linguistique, c'est le déplacement de sens du propos, avec un gain cognitif et, éventuellement, une valeur (axiologique, émotive) ajoutée. C'est pourquoi la sur-énonciation peut s'accompagner d'une incrémentation de surenchérissement qui prend en charge le PDV et marque son surcroît de pertinence : « Je suis en Savoie, et je dirais même plus, en savoir ». La sur-énonciation va de soi avec les PDV substitutifs, mais aussi avec les PDV cumulatifs puisque le cumul n'exclut pas que le PDV inattendu soit plus pertinent que le PDV attendu comme on l'a vu avec (10).

Il y a co-énonciation lorsque PDV1 et les auto- ou hétéro-commentaires expriment un accord commun, partagé, avec double prise en charge co-orientée : dans ce cas, le Lp fait l'objet d'une marque d'accord explicite (6), ou pourrait accepter l'incrémentation d'une telle marque (d'accord, tout à fait). (Mais cette co-énonciation de PDV1 va de pair avec une sur-énonciation ${ }^{36}$ envers PDV2.)

34 La différence est qu'ils prennent aussi en charge PDV2 dans leur lecture cumulative, tandis qu'avec la lecture substitutive, PDV2 est seulement pris en compte (Roulet $1981: 19$ ).

35 Voir Rabatel (2012b : 35-38) pour une définition / exemplification des trois postures énonciatives.

36 Cette double posture énonciative explique que la sur-énonciation soit moins forte que dans l'ironie (Rabatel 2012c : 64-73), l'hyperbole (Rabatel 2015a : 102-108) et la contrepèterie in absentia (Rabatel 2015b : 58, 62) et nettement différente de la sous-énonciation de l'humour 
La posture de sous-énonciation correspond au cas où L1/E1 $1^{37}$ se distancie de la formulation de PDV1, seulement prise en compte. Ainsi dans l'exemple (20') assorti du message correctif : "Merci de lire seffectivement» ", ou dans les exemples (12) et (15), même s'ils ne sont suivis d'une telle correction distanciée. En effet, lorsque PDV1 repose sur un conflit tellement hors norme qu'il peut difficilement être assumé en tant qu'énonciation sérieuse (et pas davantage comme énonciation feinte, l'énonciateur ne revendiquant pas ce registre), la distanciation n'a pas besoin d'être explicitée.

\section{Conclusion}

La logique du Lp ne repose pas sur les dysfonctionnements phonologiques ou graphologiques du système mais réside dans « l'économie énonciative qui, elle, bien évidemment se soutient des contraintes et possibilités de tel système linguistique qu'elle met en œuvre » (Fenoglio 1997 : 45). Je pourrais ajouter : des contraintes et possibilités des claviers. Si un Lp linguae relève d'une condensation primaire, le Lp clavis relève d'une condensation médiée, secondaire (voire tertiaire avec les correcteurs orthographiques !), qui repose sur les contraintes du clavier (ou des algorithmes des correcteurs), mais qui dépend, en dernière instance, de l'aptitude du résultat à faire sens d'un point de vue énonciatif / discursif. Que la logique de PDV en confrontation soit substitutive ou cumulative, elle prend la forme d'un déplacement de phonèmes/graphèmes, de syllabes phonique ou graphique, de mots et d'une condensation de $\mathrm{PDV}^{38}$, avec deux logiques discursives / énonciatives qui se télescopent.

(Rabatel 2013a : 39-41, 2013b : 106-110). Sur ces doubles postures, par rapport à des énonciateurs différents, sources de PDV distincts, voir Rabatel (2011a, 2018).

37 L'écouteur externe peut aussi être en sous-énonciation, mais uniquement pour les Lp envers lesquels il souhaite marquer une distanciation.

38 Confirmant au plan linguistique les analyses de Freud : « les lois du lapsus sont les mêmes dans toutes les langues », renvoyant à un « travail de condensation qui joue un rôle si important dans la formation des rêves » ([1901] 1969 : 111). Je n'ai pas la place de développer ce point. Pour une analyse freudienne du Lp, et, en général, d'une conception de lalangue accordant la primauté au signifiant - et à une analyse relevant, selon Lacan (1975 : 19-27), de la linguisterie, ou « grammaire du signifiant »-, voir Aquien (2016 : notamment pp. 37-43, 64-65, 91, 120-121) et supra note 31. Une précision d'importance, cependant : bien des exemples de mon corpus renvoient, comme le lecteur a pu le constater, à des événements de la vie ordinaire, notamment à des soucis, lesquels ne relèvent pas tous d'une lecture qui focalise sur des phénomènes sexuels. En ce sens, l'analyse freudienne mérite réexamen (ce constat n'est pas nouveau). J'ajoute toute- 
La notion de PDV éclaire le Lp et la prédication qui le contient (ainsi que les commentaires auto- ou hétéro-réflexifs qui l'accompagnent), puisqu'elle rend compte :

- de l'existence de deux PDV, coréférant à deux énonciateurs intratextuels distincts (autrement dit à des positions énonciatives différentes d'un même locuteur) ;

- de la nécessité d'actualiser deux prédications distinctes, signifiantes en co(n)texte, qui rétablissent la cohérence de l'énoncé condensé en privilégiant d'un côté la logique du thème, de l'autre celle du rhème ;

- de la possibilité d'interpréter les PDV selon des rapports objectivement cumulatifs ou substitutifs ou selon une interprétation cumulative ou substitutive ;

- d'expliquer que si le PDV manifeste, involontaire, est ressenti in fine comme plus pertinent que le PDV attendu, c'est parce que l'écouteur (ré)interprète le PDV involontaire et apparemment immotivé comme un PDV certes involontaire, mais motivé, intentionnel, fût-ce à un autre niveau ;

- des postures énonciatives diverses, de co-, sur- ou sous-énonciation que les énonciateurs du PDV manifeste (PDV1) (l'écouteur/locuteur et/ou les écouteurs récepteurs) entretiennent avec le PDV attendu/intenté (PDV2) ;

- du processus au terme duquel un $L p$ (clavis) peut être jugé comme bon et être considéré comme JDM, voire comme un bon JDM.

Plus largement, d'un point de vue prospectif, ce modèle - déjà mis à contribution pour l'analyse des antimétaboles (Rabatel 2008b), à-peu-près (Rabatel 2011b), hyperboles, contrepèteries, antanaclases et syllepses (Rabatel 2015a, 2015b, 2015c), mais aussi des figures de pensées comme l'ironie ou l'humour (Rabatel 2012c, 2013a) - devrait être confronté à d'autres figures (je pense notamment au paradoxe). Mais, comme j'ai eu l'occasion de le dire à maintes reprises (Rabatel 2008a), l'objectif n'est pas tant de ré-investiguer les anciennes figures de rhétorique que de s'interroger, plus largement, sur le travail de figuration et sur ses effets pragma-énonciatifs, dans le cadre général d'une approche sémantique basée sur des points de vue en confrontation (Rabatel 2018), indépendamment du fait que les énonciateurs argumentent explicitement. À terme, il s'agit de penser la complexité (notionnelle, interactionnelle, langagière) dans un cadre qui

fois que si la réinterprétation sociologique des rêves que propose Lahire (2018) conduit à prendre ses distances avec une survalorisation de la sexualité (et plus encore de la sexualité infantile), elle ne conduit pas nécessairement à rejeter dans le même mouvement les phénomènes de censure, de refoulement, ni le travail du signifiant en relation avec ce dernier. 
rende compte de l'altérité, qu'il s'agisse de formes orientées vers le sujet (les autres que soi, les autres de soi) ou de formes orientées vers l'objet à travers la complexité / altérité des référents extralinguistiques, du travail de référenciation (Danon-Boileau 1982 : 34), exploré à travers le dit et/ou le dire.

\section{Références bibliographiques}

Aquien, Michèle. 2016. Poétique et psychanalyse. L'autre versant du langage. Paris : Classiques Garnier.

Arnaud, Pierre. 1999. Target-error resemblance in French word substitution speech errors and the mental lexicon. Applied Psycholinguistics 20. 269-287.

Attardo, Salvatore. 2018. Universals in puns and humorous wordplay. In Esme Winter-Froemel \& Verena Thaler (éds.), Cultures and Traditions of Wordplay and Wordplay Research (The Dynamics of Wordplay 6), 89-109. Berlin \& Boston : De Gruyter.

Benveniste, Emile. [1947] 2015. Le jeu comme Structure. Deucalion. Cahiers de philosophie 2. 161-167. / In Emile Benveniste, Langues, cultures, religions, choix d'articles réunis par Chloé Laplantine \& Georges-Jean Pinault. 177-183. Limoges : Lambert-Lucas.

Bonhomme, Marc. 2005. Pragmatique des figures du discours. Paris : Champion.

Braun, Angelika \& Astrid Schmiedel. 2018. The phonetics of ambiguity : A study on verbal irony. In Esme Winter-Froemel \& Verena Thaler (éds.), Cultures and Traditions of Wordplay and Wordplay Research (The Dynamics of Wordplay 6), 111-136. Berlin \& Boston : De Gruyter.

Bravo, Federico. 2011. Anagrammes. Sur une hypothèse de Ferdinand de Saussure. Limoges : Lambert-Lucas.

Culioli, Antoine. 2004. De l'énonciation à la " grammaire subjective». Entretien avec Antoine Culioli. In Dominique Ducard (éd.), Entre grammaire et sens. Etudes sémiologiques et linguistiques, 7-22. Paris : Ophrys.

Culioli, Antoine. 2012. Toute théorie doit être modeste et inquiète. Entretien avec Almuth Grésillon \& Jean-Louis Lebrave. Genesis 35. 147-155.

Danon-Boileau, Laurent. 1982. Produire le fictif. Paris : Klincksieck.

Ducrot, Oswald. 1984. Le dire et le dit. Paris : Éditions de Minuit.

Fenoglio, Irène. 1997. La notion d'événement d'énonciation : le lapsus comme une donnée d'articulation entre discours et parole. Langage et société 80. 39-71.

Fenoglio, Irène. 2003. Graphie manquée, lapsus écrit : un acte d'énonciation attesté. Langage et société 103. 57-77.

Freud, Sigmund. [1901] 1969. Psychopathologie de la vie quotidienne. Lausanne : Payot.

Fromkin, Victoria. 1973. Speech Errors as Linguistic Evidence. La Haye \& Paris : Mouton.

Green, André. 2003. La cure parlante et le langage. Psychiatrie française 33(3-4), Les conférences de Lamoignon. Le langage 1. 36-61.

Halté, Pierre. 2013. Les marques modales dans les chats : étude sémiotique et pragmatique des interjections et des émoticônes dans un corpus de conversations synchrones en ligne. Thèse de doctorat, Universités de Lorraine et du Luxembourg.

Hockett, Charles F. 1984. Where the tongue slips, there slip 1. In Victoria A. Fromkin (éd.), Speech Errors as Linguistic Evidence, 93-119. La Haye \& Paris : Mouton. 
Juranville, Françoise. 2010. Lapsus et actes manquées : un nouveau regard après Freud. Journal des psychologues 277. 62-66.

Koch, Peter \& Wulf Oesterreicher. 1985. Sprache der Nähe - Sprache der Distanz. Mündlichkeit und Schriftlichkeit im Spannungsfeld von Sprachtheorie und Sprachgeschichte. Romanistisches Jahrbuch 36. 15-43.

Koch, Peter \& Wulf Oesterreicher. 2001. Gesprochene Sprache und geschriebene Sprache / Langage parlé et langage écrit. In Günter Holtus, Michael Metzeltin \& Christian Schmitt (éds.), Lexikon der Romanistischen Linguistik, Vol. I, 2, 584-627. Tübingen : Niemeyer Verlag.

Krieg-Planque, Alice. 2009. La notion de ‘formule» en analyse du discours. Besançon : Presses Universitaires de Franche-Comté.

La pensée du discours. https://penseedudiscours.hypotheses.org/ (dernière consultation le 12/01/2018).

Lacan, Jacques. 1975. Le séminaire, Livre XX : Encore (1972-1973). Paris : Éditions du Seuil.

Lahire, Bernard. 2018. L'Interprétation sociologique des rêves. Paris : Éditions de la Découverte.

Lecolle, Michelle. 2007. Polysignifiance du toponyme. Historicité du sens et interprétation en corpus. Corpus 6, http://corpus.revues.org/1122 (dernière consultation le 21/09/2015).

Lecolle, Michelle. 2016. Some Specific Insights into Wordplay Form : Sublexical vs. Lexical Level. In Sebastian Knospe, Alexander Onysko \& Maik Goth (éds.), Crossing Languages to Play with Words. Multidisciplinary perspectives (The Dynamics of Wordplay 3). 63-70. Berlin : De Gruyter.

Nemo, François. 2014. Plurisémie, intégration sémantique, sous-détermination : rendre compte des sens multiples en emploi. Etudes romanes de Brno 35(1). 41-57.

Nemo, François. 2016. Les points de vue comme strate interprétative. Corela HS 19, http://corela.revues.org/4301 (dernière consultation le 23/06/2016).

Perdue, Clive. 1980. L'analyse des erreurs : un bilan pratique. Langages 57. 87-94.

Poier-Bernhard, Astrid. 2018. Wor(l)dplay: Reflections on a writing-experience. In Esme WinterFroemel \& Verena Thaler (éds.), Cultures and Traditions of Wordplay and Wordplay Research (The Dynamics of Wordplay 6), 75-85. Berlin \& Boston : De Gruyter.

Prandi, Michele. 2016. Métonymie et synecdoque : une frontière à retracer. In Amir Biglari \& Geneviève Salvan (éds.), Figures en discours. 75-91. Paris : Académia / L'Harmattan.

Quintilien. [vers 93 apr. J.-C.] 1978. De l'institution oratoire, Livres VIII et IX. Paris : Les Belles Lettres.

Rabatel, Alain. 2008a. Figures et points de vue en confrontation. Langue française 160. 3-19.

Rabatel, Alain. 2008b. Points de vue en confrontation dans les antimétaboles PLUS et MOINS. Langue française 160. 20-35.

Rabatel, Alain. 2009. Prise en charge et imputation, ou la prise en charge à responsabilité limitée. Langue française 162. 71-87.

Rabatel, Alain. 2011a. Coconstruction interactionnelle des points de vue et variabilité des postures énonciatives au fil du discours. Être sous-énonciateur par rapport à $X$ et surénonciateur par rapport à Y. In Bertand Verine \& Catherine Détrie (éds.), L'actualisation de l'intersubjectivité en discours. Hommages à Jeanne-Marie Barbéris, 157-177. Limoges : Lambert-Lucas.

Rabatel, Alain. 2011b. Figures d'à-peu-près et nom propre. Le français moderne 79(1). 22-33.

Rabatel, Alain. 2012a. Sujets modaux, instances de prise en charge et de validation. Le Discours et la langue 3(2). 13-36. 
Rabatel, Alain. 2012b. Positions, positionnements et postures de l'énonciateur. Travaux neuchâtelois de linguistique 56. 23-42.

Rabatel, Alain. 2012c. Ironie et sur-énonciation. Vox Romanica 71. 42-76.

Rabatel, Alain. 2013a. Humour et sous-énonciation (vs ironie et sur-énonciation). L'information grammaticale 137. 36-42.

Rabatel, Alain. 2013b. Humour, sous-énonciation. In Maria Dolores Vivero-Garcia (éd.), Frontières de l'humour, 89-108. Paris : L'Harmattan.

Rabatel, Alain. 2015a. Points de vue en confrontation dans les contrepèteries. In Esme WinterFroemel \& Angelika Zirker (éds.), Enjeux du jeu des mots. Perspectives linguistiques et littéraires (The Dynamics of Wordplay 2), 31-64. La Haye : De Gruyter Mouton.

Rabatel, Alain. 2015b. Analyse pragma-énonciative des points de vue en confrontation dans les hyperboles vives : hyper-assertion et sur-énonciation. Travaux neuchâtelois de linguistique (61-62). 91-109.

Rabatel, Alain. 2015c. La plurisémie dans les syllepses et les antanaclases. Vox romanica 74. 124-156.

Rabatel, Alain. 2016a. L'énonciation problématisante : en dialogue avec Le Royaume d'Emmanuel Carrère. Arborescences : revue d'études françaises 6. 13-38, http://id.erudit.org/iderudit/1037502ar (dernière consultation le 15/07/2018).

Rabatel, Alain. 2016b. Jeux de mots, créativité verbale et / ou lexicale : des lexies et des formules. In Christine Jacquet-Pfau \& Jean-François Sablayrolles (éds.), La fabrique des mots français, 233-249. Limoges : Lambert-Lucas.

Rabatel, Alain. 2018 (sous presse). La créativité verbale dans les devinettes : points de vue cumulatifs, assertions non sérieuses et sous-énonciation. In Bettina Full \& Michelle Lecolle (éds.), Jeux de mots et créativité. Langue(s), discours et littérature (The Dynamics of Wordplay 4). Berlin \& Boston : De Gruyter.

Rastier, François. 2014. La polysémie existe-t-elle ? Quelques doutes constructifs. Études romanes de Brno 35(1). 23-39.

Reik, Theodor. 2002. Ecouter avec la troisième oreille. Paris : Bibliothèque des introuvables.

Rossi, Mario \& Evelyne Peter-Defare. 1998. Les lapsus ou comment notre fourche a langué. Paris : Presses Universitaires de France.

Roulet, Eddy. 1981. Échanges, interventions et actes de langage dans la structure de la conversation. Études de linguistique appliquée 44. 7-39.

Thaler, Verena. 2016. Varieties of Wordplay. In Sebastian Knospe, Alexander Onysko \& Maik Goth (éds.), Crossing Languages to Play with Words. Multidisciplinary perspectives (The Dynamics of Wordplay 3), 47-62. Berlin \& Boston : De Gruyter.

Winter-Froemel, Esme. 2016. Approaching Wordplay. In Sebastian Knospe, Alexander Onysko \& Maik Goth (éds.), Crossing Languages to Play with Words. Multidisciplinary perspectives (The Dynamics of Wordplay 3), 11-46. Berlin \& Boston : De Gruyter. 


\section{Annexe}

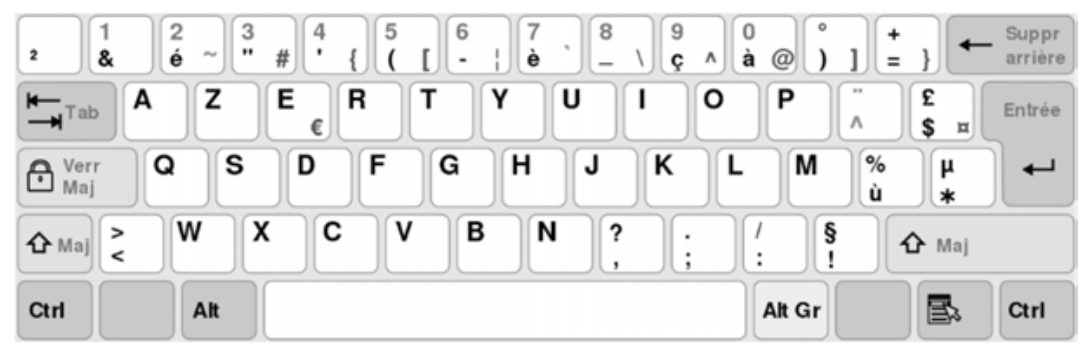

Fig. 1 : Disposition des touches sur le clavier dit «AZERTY » ${ }^{39}$

39 https://commons.wikimedia.org/wiki/File:Clavier-Azerty.svg (dernière consultation le 08/08/2018, de KB_France.svg: Yitscar [English Wikipedia], Michka B [French Wikipedia], [...] derivative work: David96 [CC-BY-SA-3.0], via Wikimedia Commons). 